\title{
Does the Wartenberg pendulum test differentiate quantitatively between spasticity and rigidity? A study in elderly stroke and Parkinsonian patients
}

\author{
R A BROWN,* DOROTHY A LAWSON, G C LESLIE, ALISON MACARTHUR, \\ W J MACLENNAN, $\dagger$ MARION E T MCMURDO, $\dagger$ W J MUTCH, $\dagger$ N J PART
}

\begin{abstract}
From the Departments of Physiology and Medicine $\dagger$ and School of Mathematical Sciences, ${ }^{*}$ The University of Dundee, Dundee, Scotland, UK
\end{abstract}

SUMMARY The results of applying the Wartenberg pendulum test to the assessment of muscle tone in populations of stroke and Parkinsonian patients are described. The test was able to distinguish between increased muscle tone of the spastic or rigid type. The Parkinsonian patients showed a marked reduction of the maximum velocity of the leg swing, with the relaxation index tending to a value of about one. In contrast the affected limb of stroke patients showed a lesser reduction of the velocity of the swing, the relaxation index tending to a value of less than one.

A simple test for the quantitative measurement of spasticity or rigidity would be valuable to both the clinician and the research worker. The Wartenberg pendulum test, ${ }^{1}$ recently re-introduced by Bajd and Vodovnik $^{2}$ is such a test and we have shown in a previous paper ${ }^{3}$ that it is suitable for use with elderly healthy subjects. In this paper we not only re-appraise the test in its computerised version ${ }^{4}$ on patients with Parkinson's disease but examine also whether the test can be extended to assess spasticity in a population of elderly stroke patients. We were anxious to establish whether it was practicable to apply the test to both sets of patients and to gain quantitative information that could relate to the underlying theoretical concepts of their conditions.

A preliminary abstract of some of these results has been presented. ${ }^{5}$

\section{Methods}

\section{Patients}

The project was approved by the local ethics committee, and all subjects freely gave their informed consent. A summary of the patient data is shown in table 1 .

Stroke patients The $\mathbf{3 0}$ subjects were all inpatients from

$\ddagger$ Present address: Department of Geriatric Medicine, City Hospital, Edinburgh, EH10 5SB, UK.

Address for reprint requests: Dr G C Leslie, Department of Physiology, The University of Dundee, Dundee, DDI 4HN, UK.

Received 27 November 1987 and in revised form 15 March 1988. Accepted 2 April 1988
Table 1 A summary of personal data from the stroke and Parkinsonian patients. For the stroke patients, the affected side refers to the side of the body showing paresis

\begin{tabular}{lll}
\hline & Females & Males \\
\hline Stroke patients & & \\
$\quad$ Total No. & 24 & 6 \\
Age (mean, SD) & $78 \cdot 0,3 \cdot 9$ & $72 \cdot 6,6 \cdot 0$ \\
$\quad$ right side affected & 9 & 3 \\
$\quad$ left side affected & 13 & 3 \\
$\quad$ affected bilaterally & 2 & 0 \\
Parkinsonian patients & & \\
Total No. & 10 & 10 \\
Age (mean, SD) & $70 \cdot 6,9 \cdot 8$ & $71 \cdot 8,4 \cdot 6$ \\
\hline
\end{tabular}

geriatric rehabilitation wards in Dundee district hospitals. All had suffered a stroke within the last 3-9 months. Only those patients whom we considered capable of sufficient relaxation during the test were selected. Prior to the pendulum test, they were given a clinical examination which included evaluation of power, tone and reflexes in the lower limbs.

Patients with Parkinson's disease Twenty subjects were entered into this group. All were ambulatory and the majority living at home. These patients prior to testing had been seen in outpatients and had been clinically assessed using the Webster ${ }^{6}$ and Hoen and $\mathrm{Yahr}^{7}$ rating scales.

\section{Recording}

The experimental methods used in these investigations were similar to those described previously, ${ }^{3}$ that is the movement of the lower leg swinging under the action of gravity was recorded and analysed. However, in contrast to a previous investigation on healthy elderly people who were tested in three positions, lying, seated and an intermediate semisupine position with the back supported at an angle of $30^{\circ}$ 

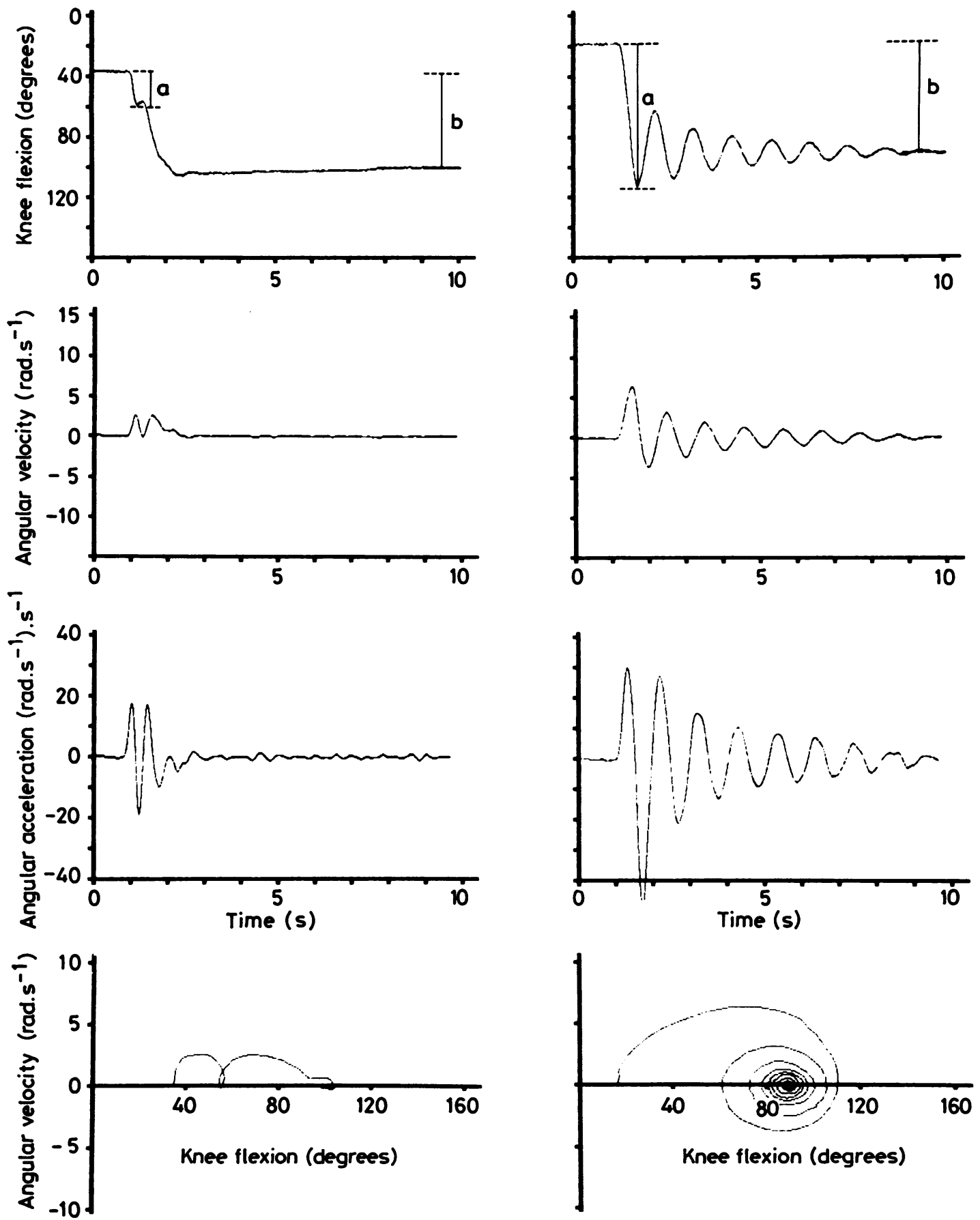

Fig 1 Eight computer print-outs from two pendulum tests on a totally relaxed and cooperative stroke patient. Records on the left of the figure were from the test on the patient's left leg, which was paretic and somewhat spastic. Records on the right were from the "unaffected" right leg. The uppermost pair of records display the angular changes occurring about the respective knee joints during oscillations following release of the subject's leg. The bar marked " $a$ " indicates the amplitude of the first swing, the bar marked " $b$ " the difference in angle between the start and end positions of the limb. The relaxation index (ratio, R2), defined by Bowman and Bajd, ${ }^{8}$ is $a / b$, and in this case was 0.355 for the spastic leg and 1.532 for the other. Note the differences in the maximum values of velocity, acceleration and rebound acceleration on the first swings of the two limbs, the spastic limb again having the smaller values of these respective measures. The lowermost records are the "phase (plane) plots", obtained by plotting the velocity data against the angle data. This form of display shows up with remarkable ease the totally different forms produced by spastic limbs as opposed to the normal type of "whirlpool" presentation associated with a healthy person's response (see ref 3 , fig 1 ). 
to the horizontal, patients in the present study were all investigated in the semi-supine position.

The recordings on the stroke patients were made, prior to their physiotherapy session, in a sideroom at the geriatric Rehabilitation Unit. Patients with Parkinson's disease attended in the afternoon for investigation, at the Department of Physiology, some 2 hours after their last dose of medication.

\section{Data handling and presentation}

The data were entered into the University mainframe computer (DEC 10) and then submitted to analysis described in the previous paper. ${ }^{3}$ The data were tested statistically with the paired $t$ test, the two sample $t$ test or the Fisher-Behrens test, as appropriate.

As in the previous paper ${ }^{3}$ some of the data are presented in the form of "Box and Whisker" plots. Such presentations, by their markers, provide five items of data. The box indicates the middle $50 \%$ of the data values between the upper and lower quartiles, the line within the box is the median value and the "whiskers" extend to the extremes of the range. Thus one may visualise the overall range, the nature of the distribution, with a measure of its variability and any tendency to skewness.

\section{Results}

The results are presented in three sections, first those

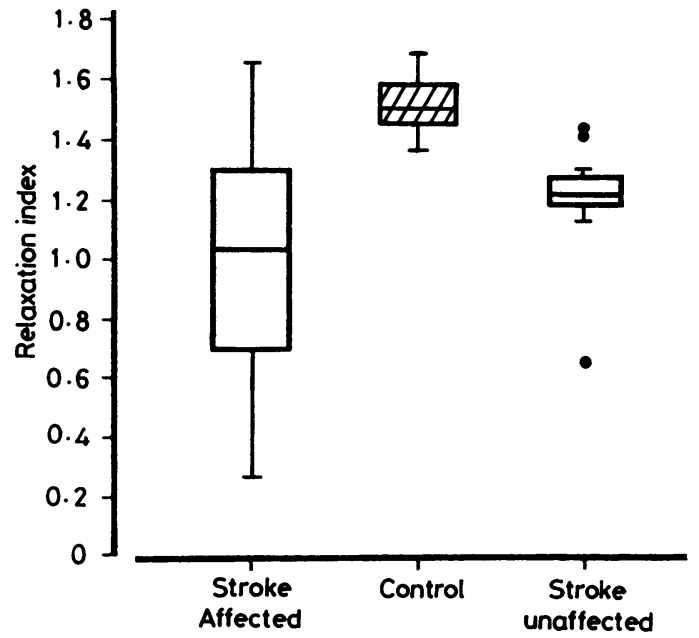

Fig 2 A composite series of "Box and Whisker" plots summarising data on the relaxation index for the affected $(n=27)$ and unaffected $(n=15)$ limbs of stroke patients and a control group $(n=30)$ whose results were reported in the preceding paper. ${ }^{3}$ Each box incorporates the central $50 \%$ of the values in the distribution, the bar within the box indicates the median value, and the outer bars mark the extremes of the distributions. In the case of the unaffected stroke patient's data there were three values which lay outside the $95 \%$ limits of the distribution and these have been expressed individually as points. from the stroke patients, second those from the patients with Parkinson's disease, and finally a comparison between these two sets of data.

\section{Stroke patients}

The first step in conducting the pendulum test was to lift the lower leg to the horizontal position. Only if the full weight of the limb was borne by the investigator was it then considered that the patient was satisfactorily relaxed. Fifteen of the patients were judged by this criterion to have relaxed their leg sufficiently for the test to give meaningful data from both legs. Twelve of the patients relaxed the affected leg but not the unaffected leg and three patients were not able to relax either leg sufficiently for the data obtained to be included in the results. Therefore this paper includes the data from the affected leg of 27 patients in total but where comparisons of the data from the affected and unaffected legs are presented the data have been obtained from 15 patients.

Examples of experimental records obtained from a spastic stroke patient who was able to relax both affected and unaffected leg are shown in fig 1 . It is immediately apparent from these records that the swing patterns of the two legs are quite different. The right, unaffected, side gives records which are similar to those previously recorded from normal healthy control subjects. ${ }^{3}$ The record from the left, affected side, differs markedly. The most obvious difference between these records is that the unaffected leg overshoots the final resting position whereas the affected leg does not. The shapes of the downswing traces also differ on the two sides; the unaffected leg produces a smooth trace without the inflection seen on the trace from the affected side. This inflection is the characteristic feature of the trace from a spastic limb. The relaxation index, also referred to as $\mathrm{R} 2$, has been used as a means of quantifying the spasticity from a record of a pendulum swing by Bajd and Vodovnik ${ }^{2}$ and this index was also measured from the records of the elderly normal subjects. ${ }^{3}$ For the normal subject the relaxation index (R2) is calculated as the ratio between the angle difference of the first swing and the difference in angle between the initial position and the final resting position (see ref 3 , fig 1 ). For records from spastic limbs showing an inflection, the relaxation index was calculated as the ratio of the angle change to the point of inflection and the difference in angle between the start and rest positions. Thus the record from the spastic leg shown in fig 1 shows a start angle of $37^{\circ}$, and angle at the inflection of $59^{\circ}$, and a final angle of $100^{\circ}$. From this data the relaxation index is calculated as $(59-37) /(100-37)=0.35$.

The distribution of the relaxation indices recorded from the affected legs of 27 patients is shown in the left hand box plot of fig 2 . The mean of these data is 0.99 , 
SD 0.41 . In comparison the mean relaxation index of 1.54 , SD 0.09 for healthy elderly subjects in the same semi-supine position ${ }^{3}$ was significantly higher $(p<0.001)$. Data from the unaffected legs of the 15 subjects whose results were usable gave a mean relaxation index of $1 \cdot 21$, SD $0 \cdot 17$; this value was also significantly different $(p<0.001)$ from the control mean of 1.54 given above.

The relaxation indices for the 15 subjects, from whom records from both sides could be obtained, are shown in fig 3 . For 11 of the subjects the relaxation index was less on the affected side than on the unaffected side but for four the ratio was greater for the affected side than for the unaffected side; this will be further considered in the discussion.

The box plots of fig 4 show the distributions of the

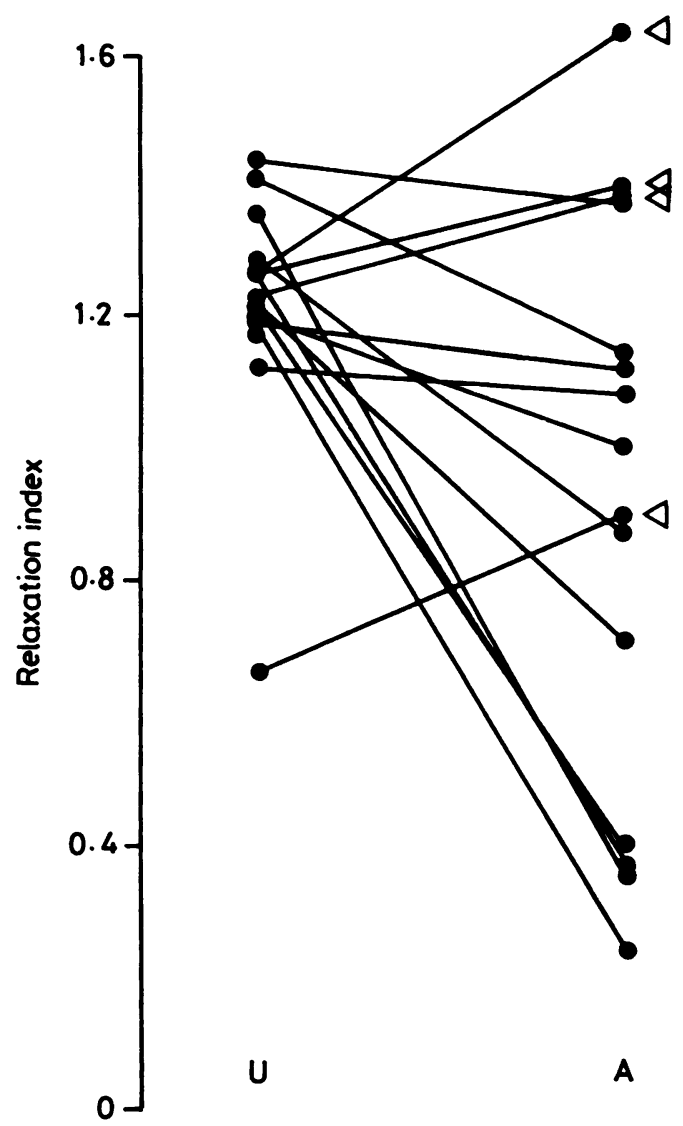

Fig 3 The relationship between the relaxation index from the affected $(A)$ and unaffected $(U)$ legs of the 15 stroke patients in whom it was possible to obtain meaningful data from both limbs. Individual's data points are joined by line. In four patients the relaxation index on their affected side was greater than that on the unaffected side and these data are indicated $(\triangleleft)$.

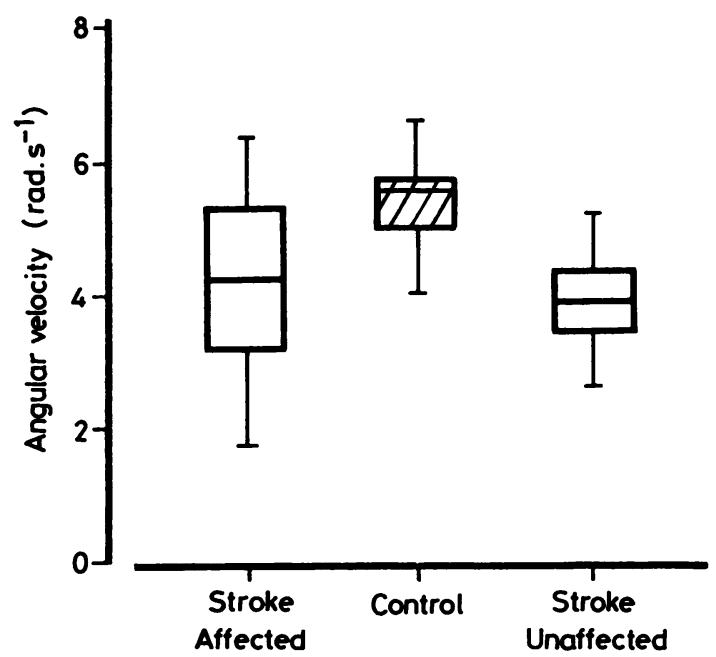

Fig 4 A composite series of "Box and Whisker" plots summarising data on the maximum angular velocity on the first swing for the affected $(n=27)$ and unaffected $(n=15)$ limbs of stroke patients and a control group $(n=30)$ whose results have been reported previously. ${ }^{3}$ The boxes are as detailed in fig 2 . The distribution of this measure is tighter for the unaffected limb in stroke patients, while both distributions from the stroke patients have medians somewhat equal but less than that for the control group.

maximum velocities reached during the initial swing for the affected (left hand box) and unaffected (right hand box) sides. The mean velocities from the affected and unaffected sides were the same at $4.05 \mathrm{rad}^{-1} \mathrm{~s}^{-1}$ but were significantly lower $(p<0.001)$ than that from elderly healthy people (5.62 rad.s $\left.\mathrm{s}^{-1}\right)$. Comparisons of the velocities from the two legs from these 15 patients are shown in fig 5. It is apparent that there is no systematic difference in velocity between the affected and the unaffected side.

The angle data were also used to calculate the maximum acceleration during the initial downswing and the rebound (table 2).

Systematic longitudinal studies of individual stroke patients were not attempted in this project. However data were obtained from one patient at an interval of 13 weeks. The mean values for relaxation index obtained on the two occasions from the affected leg showed an increase from 0.701 to $1 \cdot 21$. This patient had been undergoing regular sessions of physiotherapy throughout the interval betwen the two recordings.

\section{Patients with Parkinson's disease}

Figure 6 shows records of angle and velocity data from two patients with Parkinson's disease. The records on the left are from a patient little affected 


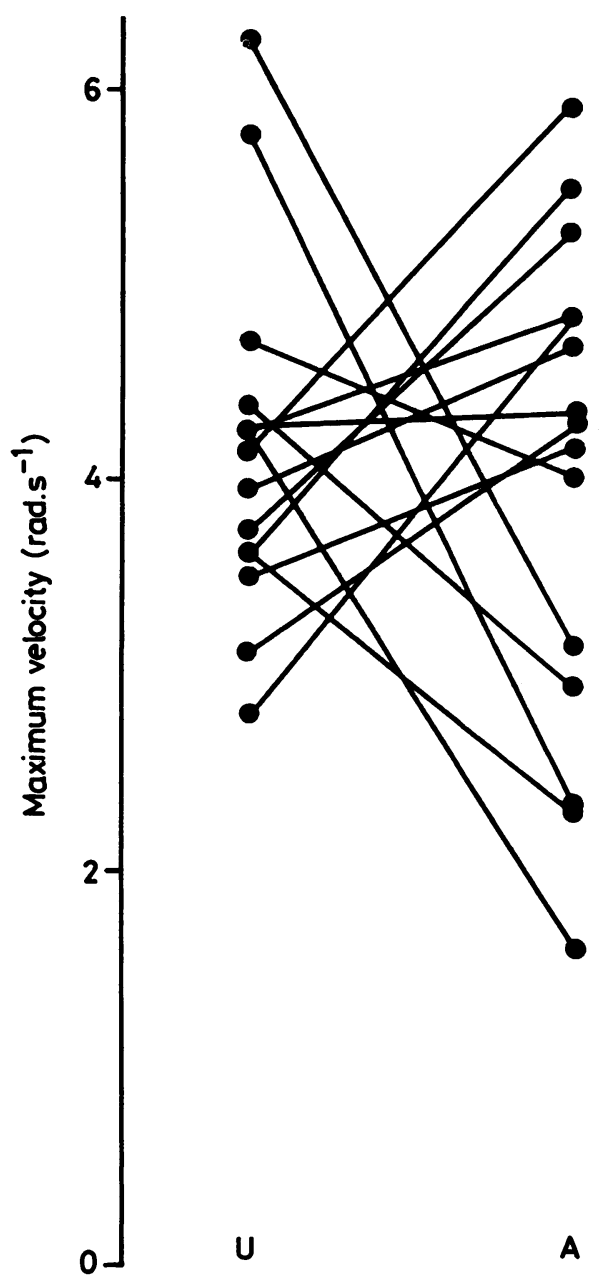

Fig 5 The relationship between maximum angular velocity from the affected $(A)$ and unaffected $(U)$ legs of the 15 stroke patients in whom it was possible to obtain meaningful data from both limbs. Individual's data points are joined by line.

with rigidity, whilst those on the right show a typical set of traces from a patient with marked rigidity. Points worthy of note are the reduced number of oscillations of the leg of the patient with mild rigidity as compared with that of a normal control subject and the complete lack of any oscillations for the patient with marked rigidity.

Collected data on the relaxation indices from both legs, of the 20 patients irrespective of sex, are shown in the box plots of fig 7 . It can be clearly appreciated that there is little difference in this measure between legs, and that for the majority of these patients their relaxation indices are below those of normal subjects (see reference 3, fig 3). Figure 8 shows that there was also a reduction for the maximum velocity on first swing in patients with Parkinson's disease. The data for the acceleration of the first and rebound swings are given in table 2.

A comparison between the data from patients with a stroke and with Parkinson's disease

An informative way of presenting the data visually is to plot the maximum velocity of the first swing against the relaxation index R2. Figure 9 shows such plots of the data from the Parkinsonian patients and from both the affected and unaffected legs of the stroke patients. Also shown on each of the plots is the convex hull of the data from the control subjects in the semisupine position (see reference 3, fig 4); the convex hull of a group of data points is formed by drawing a line connecting the outermost data points. These plots reveal that the data from the patient groups splay out from the position of the control data. The data from the unaffected legs of the stroke patients are with the exception of a single point, not far outwith the convex hull of the relevant normal control group, whereas the data from the affected legs stretches from the control data towards the $y$-axis. In contrast the data from both legs of the Parkinsonian patients stretch from the control data towards the $x$-axis. These plots restate the differences between the spastic and Parkinsonian patients that are evident from the experimental records of figs 1 and 6. With increased severity of Parkinsonian rigidity there is a decrease in the velocity of the leg swing associated with the relaxation index tending towards 1 . However with increasing severity of spasticity there is tendency for the relaxation index to be reduced to below 1; this reduction is associated with a smaller reduction in velocity of swing than is seen with the Parkinsonian patients.

Table 2 Data on the acceleration and rebound acceleration of the first swing in the stroke and Parkinsonian patients, with the data from normal healthy subjects

\begin{tabular}{|c|c|c|}
\hline & Acceleration & $\begin{array}{l}\text { Rebound } \\
\text { Acceleration } \\
\left.{ }^{-1}\right) . s^{-1}\end{array}$ \\
\hline $\begin{array}{l}\text { Stroke patients } \\
\text { Affected side } \\
\text { Unaffected side }\end{array}$ & $\begin{array}{l}23 \cdot 1, \text { SD } 7 \cdot 1 \\
17 \cdot 0, \text { SD } 4 \cdot 6\end{array}$ & $\begin{array}{l}-31 \cdot 9, \text { SD } 10 \cdot 8 \\
-25 \cdot 9, \text { SD } 8 \cdot 8\end{array}$ \\
\hline $\begin{array}{l}\text { Parkinsonian patients } \\
\text { Left side } \\
\text { Right side }\end{array}$ & $\begin{array}{l}14 \cdot 3, \text { SD } 14 \cdot 1 \\
12 \cdot 4, \text { SD } 6 \cdot 2\end{array}$ & $\begin{array}{l}-19 \cdot 1, \text { SD } 18.6 \\
-18 \cdot 8, \text { SD } 10.9\end{array}$ \\
\hline $\begin{array}{l}\text { Normal controls* } \\
\text { Left leg } \\
\text { Right leg }\end{array}$ & $\begin{array}{l}24 \cdot 5, \text { SD } 4 \cdot 1 \\
27 \cdot 7, \text { SD } 4 \cdot 9\end{array}$ & $\begin{array}{l}-41 \cdot 8 \text {, SD } 7 \cdot 9 \\
-43 \cdot 3 \text {, SD } 8 \cdot 0\end{array}$ \\
\hline
\end{tabular}
*These data have been carried over from table 2 in the preceding
paper. $^{3}$ 

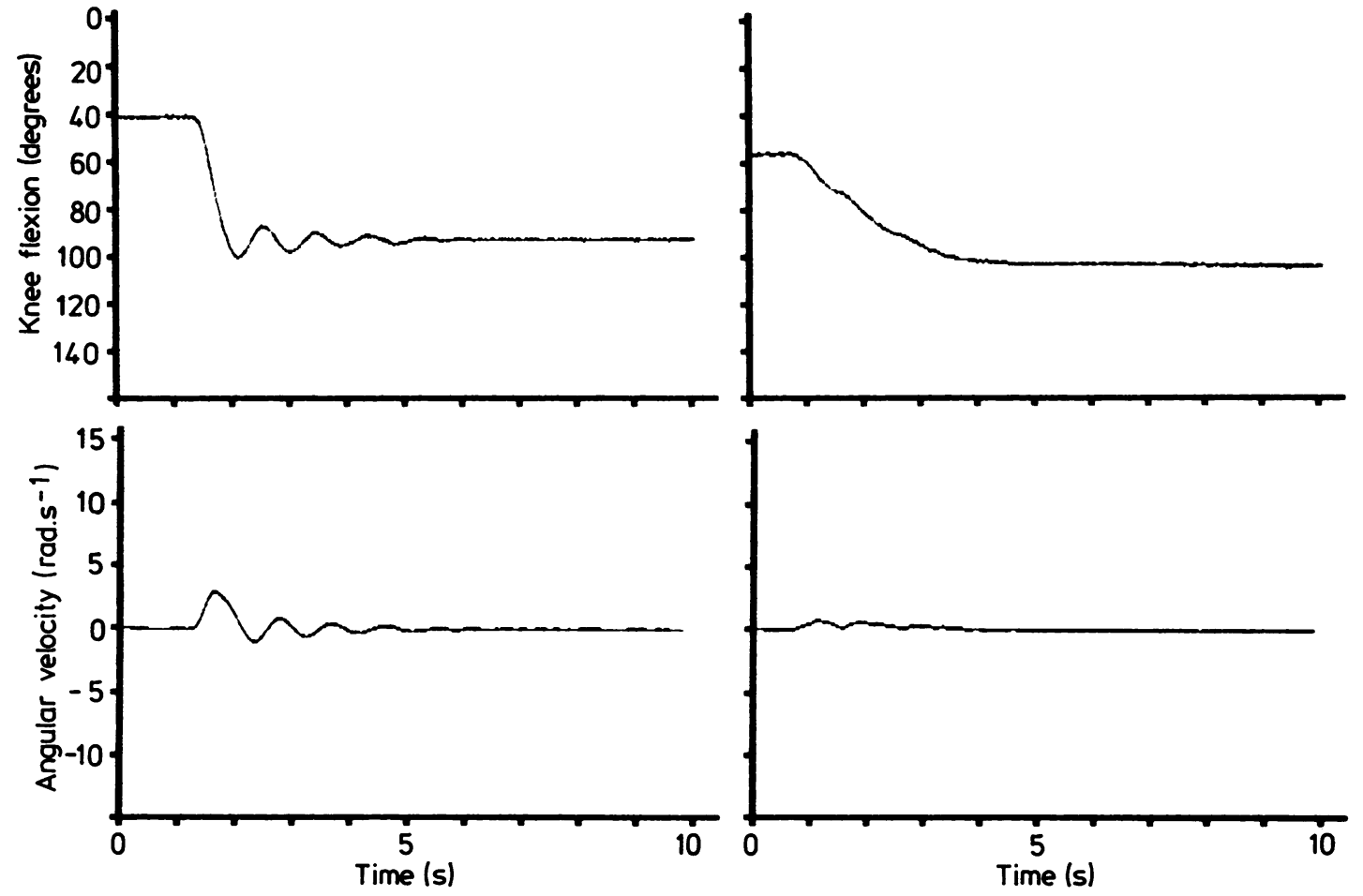

Fig 6 Computer print-outs from pendulum tests on two patients with Parkinson's disease. Records on the left were from a patient with only moderate rigidity, those on the right from a patient severely affected. Note the reduced number of swings, as compared with healthy normals, in the moderately affected patient and the total absence of swings in the severely affected patient, also the reduced quantified velocity of movements of the limbs in these two patients.

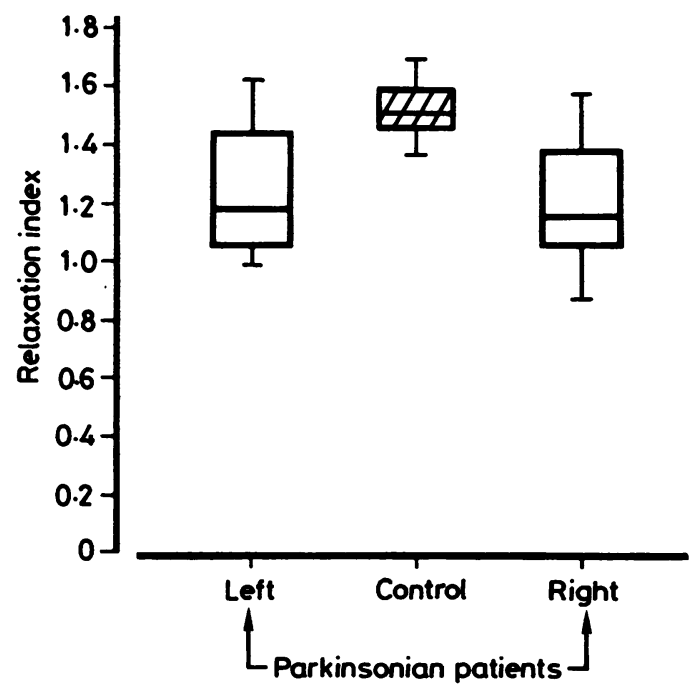

Fig 7 A composite series of "Box and Whisker" plots summarising data on the relaxation index for the left and right legs of 20 patients with Parkinson's disease, together with comparable data from a control group $(n=30)$ whose results were reported in the preceding paper. ${ }^{3}$ Clearly the majority of values of relaxation index in the distributions from the patients is less than that in healthy controls.

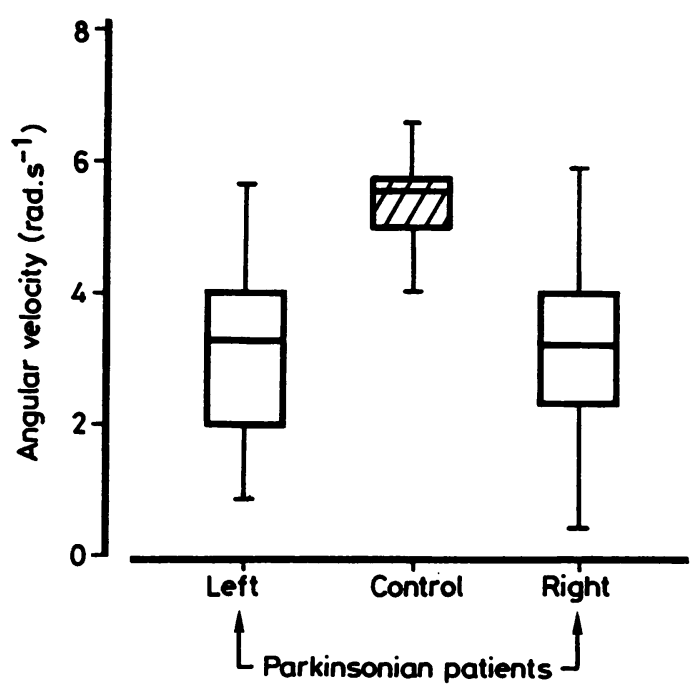

Fig 8 A composite series of "Box and Whisker" plots summarising data on the maximum angular velocity on first swing in pendulum tests on the left and right legs of 20 patients with Parkinson's disease, together with comparable data from a control group $(n=30)$ whose results were reported in the preceding paper. ${ }^{3}$ 


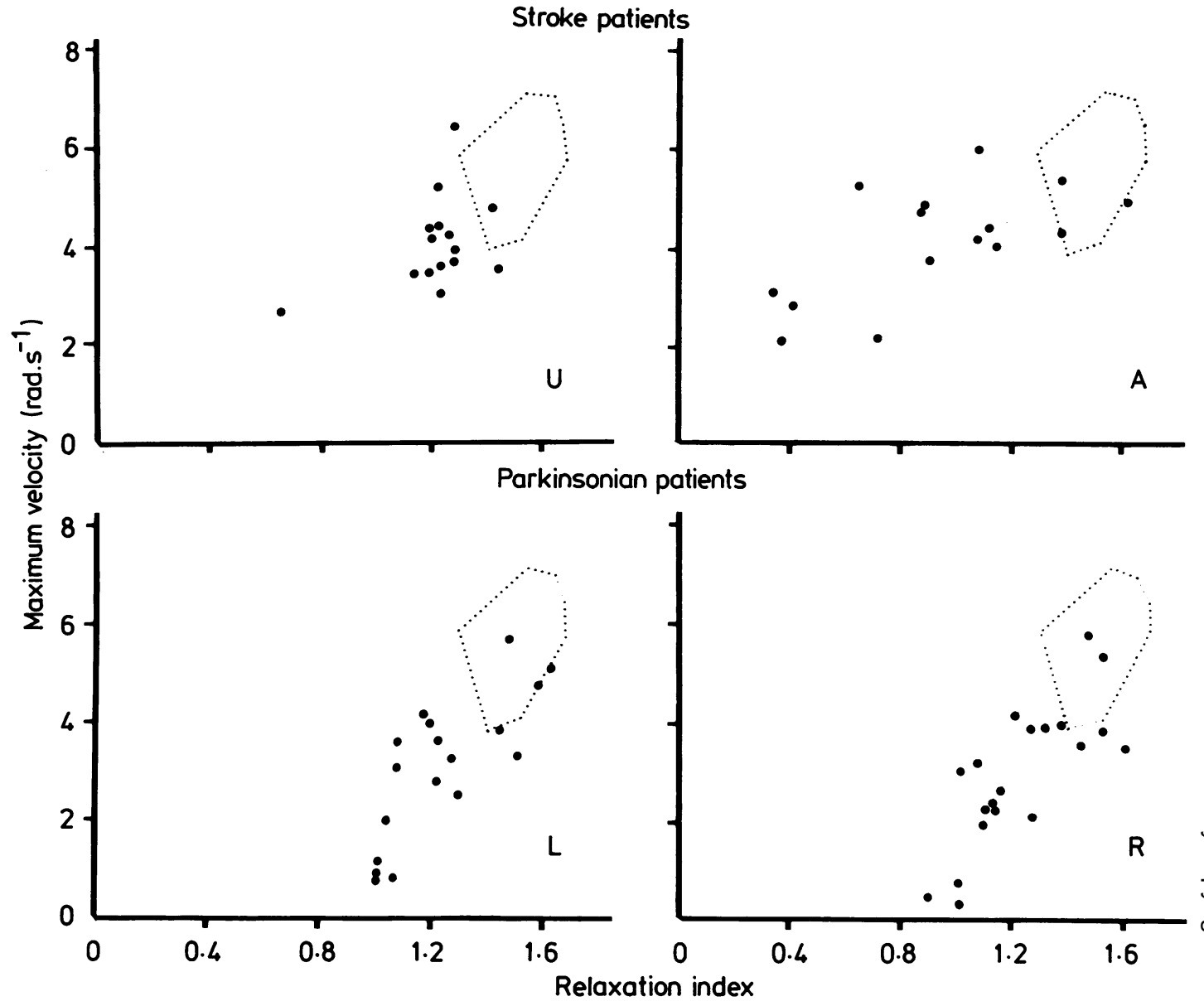

Fig 9 The relationship between relaxation index and maximum velocity on first swing for the data from 15 stroke patients (upper part of figure, $U=$ unaffected limb, $A=$ affected, paretic limb)) and 20 patients with Parkinson's disease (lower part of figure, $L=$ left leg, $R=$ right leg). Within each quadrant there is also shown the convex hull encompassing the corresponding data of a group of 30 elderly healthy control subjects and who had been investigated in the same semi-supine position as had been adopted by the patients when tested. The convex hull allows easy appreciation of how much a patient's data diverge from normal.

\section{Discussion}

The traditional clinical method for the assessment of muscle tone involves estimation of the resistance to manipulation of the limb by the investigator. Voluntary contraction of that limb during the estimation hinders accurate determination of muscle tone. The same problem is encountered when attempting to determine the tone by means of the Wartenberg pendulum test, a point stressed by Wartenberg in his original description. ${ }^{1}$ It was our experience that it was often those subjects who were not able to relax sufficiently for the pendulum test who also presented difficulties for the traditional method. The sensation of the leg swing, without any input from the subject, is a strange one to which even a healthy subject takes a little time to become accustomed. Although the test was not unpleasant, our elderly patients, and in particular the stroke patients, were often anxious about it, so that they had difficulty in relaxing during the pendular movement of their leg. Ways of minimising the problem are to select only patients with sufficient cognitive ability to be cooperative and to spend a considerable amount of time reassuring and distracting those selected. With these provisos, it was possible to obtain useful data with the pendulum test. Even so, only about half of the stroke patients were able to relax sufficiently for the test to be applied 
successfully to both the affected and the unaffected side. However, if data are required only from the affected side then there is not the same problem with patient selection because many stroke patients have insufficient control over the affected side to enable them actively to interfere with the passive swing pattern. Wartenberg ${ }^{1}$ came to similar conclusions in his original study. Far less difficulty was experienced in putting the Parkinsonian patients into a sufficiently relaxed state to perform the test. This probably reflects on the reasonably normal life in the community led by the Parkinsonian patients as opposed to that of the stroke patients, who were resident in a rehabilitation unit.

An explanation of the differences in the data obtained from the stroke and Parkinsonian patients requires consideration of the principles underlying the test. On release from the horizontal position the lower leg is subjected to angular acceleration. In a spastic limb a stretch induced contraction of the muscle is brought about at stretch velocities of between $8.70 \times 10^{-2} \mathrm{rad}_{\mathrm{s}} \mathrm{s}^{-1}$ and $3.4 \mathrm{rad} . \mathrm{s}^{-1} .9$ The mean value of the maximum velocity during the downswing in normal subjects was in excess of 4 rad.s ${ }^{-1}$ (see reference 3 , table 2 ), that is a greater velocity than that required to activate a spastic limb. Therefore a spastic limb will reach under test a velocity sufficiently great to evoke a reflex response. This is the cause of the inflection seen in the trace obtained from the spastic limb illustrated in fig 1 . The greater the velocity the sooner in the trace the inflection caused by spasticity would be expected and hence a lesser value for the relaxation index. The mean value for the relaxation index is indeed significantly less than that obtained from control subjects tested in the same position.

The recorded value of the relaxation index was as low as about 0.3 in some of the spastic patients whereas the relaxation index recorded from the Parkinsonian patients was not less than about 1 . This indicates that Parkinsonian patients did not show the velocity dependent muscle activation shown by the spastic patients. A relaxation index of about 1 means that there is no overshoot of the final resting position; the rigidity of the leg produces such a degree of damping that the swing stops on the first swing at the final resting position.

The data from the stroke patients shows that not only is the value of relaxation index from the leg with abnormal clinical signs significantly different from the controls, the value recorded from the apparently normal legs of the stroke patients is also significantly different. This could be a result of either a lack of complete relaxation at the moment of the test or bilateral involvement not detected by standard clinical techniques. Great care was taken to ensure that the subject was as well relaxed as possible and results were rejected from all subjects who did not fit the criterion described in Results. It is likely therefore that the clinically "unaffected" side was in fact affected. Possibilities are that the test is sensitive enough to detect the effect of a cerebrovascular accident on uncrossed nerve fibres of the corticospinal tract, ${ }^{10}$ or that changes are produced bilaterally at the segmental spinal level as a result of the altered functions of the affected side.

In 11 out of the 15 stroke patients in whom both sides could be tested satisfactorily the value for the relaxation index was less on the affected than on the unaffected side. More surprisingly, in four of the stroke patients (see marked data in fig 3 ) the value from the unaffected side was less than that from the affected side. Three of these patients had relatively high values of relaxation indices and did not show any marked degree of spasticity on either side and the fourth patient had been found on clinical examination to be somewhat spastic on both sides. It may be that the four patients had multiple cerebral lesions affecting both cerebral hemispheres but that clinical evaluations failed to identify this. In future studies computed tomography of the head, both immediately after the stroke and prior to testing, might be useful in investigating this point.

The maximum velocity during the initial swing was significantly less than that recorded from the control subjects for both the stroke patients and the Parkinsonian patients. In the stroke patients, there was no systematic difference between the maximum velocity recorded from the affected and unaffected legs, a finding also reflected in the similarity of the means of the data from the affected and unaffected sides. The velocity of the swing could be affected by a number of factors such as the mechanical properties of the knee joint and quadriceps muscle, and also of course any reflex activity in that muscle. In the case of spastic as opposed to rigid muscle such reflex activity would not come into effect until the velocity of the swing is sufficient to activate the stretch reflex. This is evident from fig 9 in which it is apparent that as the data points move out from the area control data there is a very much smaller reduction in velocity than is the case with the Parkinsonian patients' data points. The lower leg of the Parkinsonian patients swings down at lower velocity than does that of the affected side of the stroke patients. The velocity in the former is too low to bring about any reflex activation of the quadriceps, a fact evident from the lack of an inflection on the traces from the Parkinsonian patients. These differences in relaxation index and velocity between stroke and Parkinsonian patients account for the distinctive difference between the velocity/index plots of fig 9 for the two different types of patient.

The question arises as to the clinical application of 
the pendulum test. Firstly Wartenberg ${ }^{1}$ was in no doubt as to the test's importance "in alerting the examiner (to) possible early organic disturbances". Our present, limited, experience of the computerised test on normal subjects and neurological patients does not allow a definitive statement on its applicability as a quantitative means of presymptomatic diagnosis of rigidity. It is exciting nonetheless to speculate that, with further study, the test might detect those in the population harbouring sub-clinic Lewy body pathology of their pigmented brain stem nuclei. Secondly, and speculation apart, the test does provide an additional means, beyond the traditional clinical tests, of distinguishing between spasticity and rigidity (see fig 9). Thirdly this simple method of obtaining quantitative data provides the opportunity to follow the development of either spasticity or rigidity. This would be of particular value in following the natural history of muscle tone following a stroke or the first (speculative, see above) diagnosis of Parkinson's disease. In addition quantitative assessment of the effects of drug treatment and/or physiotherapy on spasticity and rigidity could also be obtained.

We thank all patients for their cooperation in this investigation, and Dr B Andrews and his colleagues at the Department of Bioengineering, University of Strathclyde, Glasgow, for generously making available their software package. Both Dr Andrews and ourselves (GCL/NJP) are willing to discuss availablity of our current software packages with anyone interested.

This work was in part financially supported by Norwich Eaton. We thank Mr J Winslade of Norwich Eaton for his continuing interest in our research.

\section{References}

1 Wartenberg R. Pendulousness of the legs as a diagnostic test. Neurology 1951;1:18-24.

2 Bajd T, Vodovnik L. Pendulum testing of spasticity. J Biomed Eng 1984;6:9-16.

3 Brown RA, Lawson DA, Leslie GC, Part NJ. Observations on the applicability of the Wartenberg pendulum test to healthy, elderly subjects. $J$ Neurol Neurosurg Psychiatry 1988;51:1171-77.

4 Mulvey N, Quinn J, Andrews BJ, Bajd T. A microcomputer based instrument for pendulum testing of spasticity. Dem:19. In: Jordan MM, Perkins WJ, eds. Computed-aided Biomedical Imaging and Graphics Physiological Measurement and Control. London: The Biological Engineering Society 1984.

5 Leslie GC, MacArthur A, Mutch WJ, Part NJ. A preliminary and quantitative reappraisal of the Wartenberg test in Parkinsonian patients. Proceedings of an international symposium on "Mechanoreceptors: Development, structure and function. Prague, August 11-15, 1987. New York: Plenum (in press).

6 Webster DD. Critical analysis of the disability in Parkinson's disease. Modern Treatment 1968;5: 257-82.

7 Hoehn MH, Yahr MD. Parkinsonism: Onset, Progression and Mortality. Neurology 1967;17:427-42.

8 Bowman B, Bajd T. Influence of electrical stimulation on skeletal muscle spasticity. In: Popovic D, ed. Proceedings of the Seventh International Symposium on External Control of Human Extremities. Belgrade: Yugoslav Committee for Electronics and Automation, 1981:567-76.

9 Burke D, Gillies JD, Lance JW. The quadriceps stretch reflex in human spasticity. $J$ Neurol Neurosurg Psychiatry 1970;33:216-223.

10 Carpenter MB, Sutin J. Human Neuroanatomy. Baltimore: Williams \& Wilkins, 1983:8th ed. 282-4. 